Rev. Elev. Méd. Vét. Pays trop., 1979, 32 (3) : 285-290.

\title{
Caractéristiques de la reproduction de la brebis oudah
}

\author{
par Y. GAILLARD $\left({ }^{*}\right)$
}

\begin{abstract}
RÉSUME
L'étude des caractéristiques de la reproduction du mouton Oudah a été entreprise sur un petit troupeau à l'Université de Niamey, de janvier 1977 à juin 1979. Le comportement sexuel de la femelle est décrit, ainsi que les principaux résultats ayant trait à l'ostrus (longueur du cycle, durée de l'cestrus, répartition annuelle), à la durée de la gestation et au nombre de petits par portée.
\end{abstract}

\section{INTRODUCTION}

Les moutons à poils représentent, après les zébus, la principale richesse de l'élevage nomade sahélien au Niger.

Les moutons à poils du Niger sont représentés par 2 races, la race targui et la race peulh. La race peulh possède 2 variétés principales: le Bali-bali qui peuple principalement le nord du pays jusqu'à l'isohyète $600 \mathrm{~mm}$, et le Oudah plus au sud (2).

Ces moutons peulh sont caractérisés par leur grande taille, une robe bicolore (avant-train foncé noir ou fauve, arrière-train blanc) et par les cornes horizontales du bélier, ainsi que de très longues oreilles.

Malgré leur importance économique considérable, aucune étude zootechnique, en particulier sur la reproduction, n'avait encore été entreprise.

\section{MATÉRIEL ET MÉTHODES}

Du mois de janvier 1977 au mois de juin 1979, nous avons effectué des observations suivies ainsi que différentes expériences sur un petit troupeau de moutons Oudah entretenus à l'Université de Niamey, Niger.

(*) D-8069, Tegernbach, Waldweg 12, Allemagne.
Les animaux sont nourris à base de Bourghou (Echinochloa stagnina, de feuilles et de tiges de Niébé (Vigna sinensis), de son de riz et d'un supplément minéral.

Le troupeau était constitué au départ de 9 femelles âgées de 2 à 6 ans et d'un bélier de 4 ans. Une partie des jeunes issus du troupeau fut conservée, si bien que nous disposions, en 1979, de 14 femelles et 5 mâles sexuellement mûrs et de 9 jeunes de moins de 8 mois.

Les principales caractéristiques zootechniques furent étudiées : croissance, fertilité, caractéristiques de la physiologie sexuelle. Depuis janvier 1977, des contrôles journaliers et des périodes d'observations de longue durée (journée ou nuit) furent effectuées, au total $408 \mathrm{~h}$ d'observations. Pendant certaines périodes, les femelles étaient séparées des mâles et soumises 2 à 3 fois par jour $(8,13$ et $18 \mathrm{~h})$ à un contrôle d'oestrus. On utilisait pour cela un bélier muni d'un tablier empêchant l'intromission. L'œstrus est défini comme la durée entre la première et la dernière monte acceptées par une brebis.

Différentes expériences ont été réalisées afin de tester les performances sexuelles des animaux ainsi que l'influence de différents facteurs sur les caractéristiques de l'cestrus.

Un second groupe de 5 femelles sur lesquelles A. YENIKOYE étudie les variations du taux de progestérone au cours du cycle astrien est soumis à 2 contrôles d'œstrus journaliers (18). 


\section{LE COMPORTEMENT SEXUEL}

De même que chez les moutons à laine européens $(3,13,15)$, le comportement sexuel des brebis Oudah est très discret et ne s'extériorise généralement què pendant l'œstrus et en présence d'un bélier.

\section{Extériorisation des chaleurs sans bélier présent}

Une légère augmentation de la nervosité peut être notée : l'irritabilité et les déplacements s'intensifient. Les montes entre femelles, signe typique de l'œstrus chez les bovins domestiques, sont ici très rares. Je n'en ai observées qu'à 4 reprises dont 3 en absence de mâle. La rareté de ces montes est aussi notée chez les brebis à laine européennes (15). La position immobile debout ávec la tête penchée vers le sol qui est parfois décrite comme typique de l'œstrus chez les brebis européennes (MATTNER et al., 1967, GRANT, 1934 et CORDTS, 1953 dans SAMBRAUS, 1973 (15) n'est jamais ici en relation avec l'œstrus : cette posture s'observe fréquemment, aussi bien chez les béliers que chez les brebis en cstrus ou non ; en général elle est adoptée par des animaux fatigués, surtout lorsqu'il fait très chaud.

Dans la pratique, seule la présence d'un béher permettra de détecter un cestrus.

\section{Comportement de la brebis en présence d'un bélier}

Brebis en diastrus: les femelles en diœstrus répondent en général aux avances ou aux contrôles génitaux du mâle en urinant, puis en s'enfuyant si ce dernier insiste (Photo I).

Brebis en procestrus : un bélier expérimenté peut détecter 1 à 3 jours avant une femelle qui va être en cestrus. Il effectue alors des contrôles génitaux et des poursuites de plus en plus fréquents envers cette femelle. Pendant cette période, la brebis se dérobe énergiquement aux approches du mâle en frétillant beaucoup de la queue.

Brebis en astrus, parade sexuelle: l'œstrus comportemental est défini comme la période entre la première et la dernière monte acceptées par une femelle. Nous ne ferons ici que décrire le comportement typique de la brebis pendant la parade sexuelle ; ce comportement est en fait à considérer dans une chaîne réactionnelle entre mâle et femelle, ce que nous ferons plus précisément dans un article consacré au bélier.

Lorsque l'œstrus s'installe, la femelle va participer activement à la cérémonie précopulatoire :

Dans un premier stade la brebis s'enfuit vivement à toute approche du bélier, décrivant de

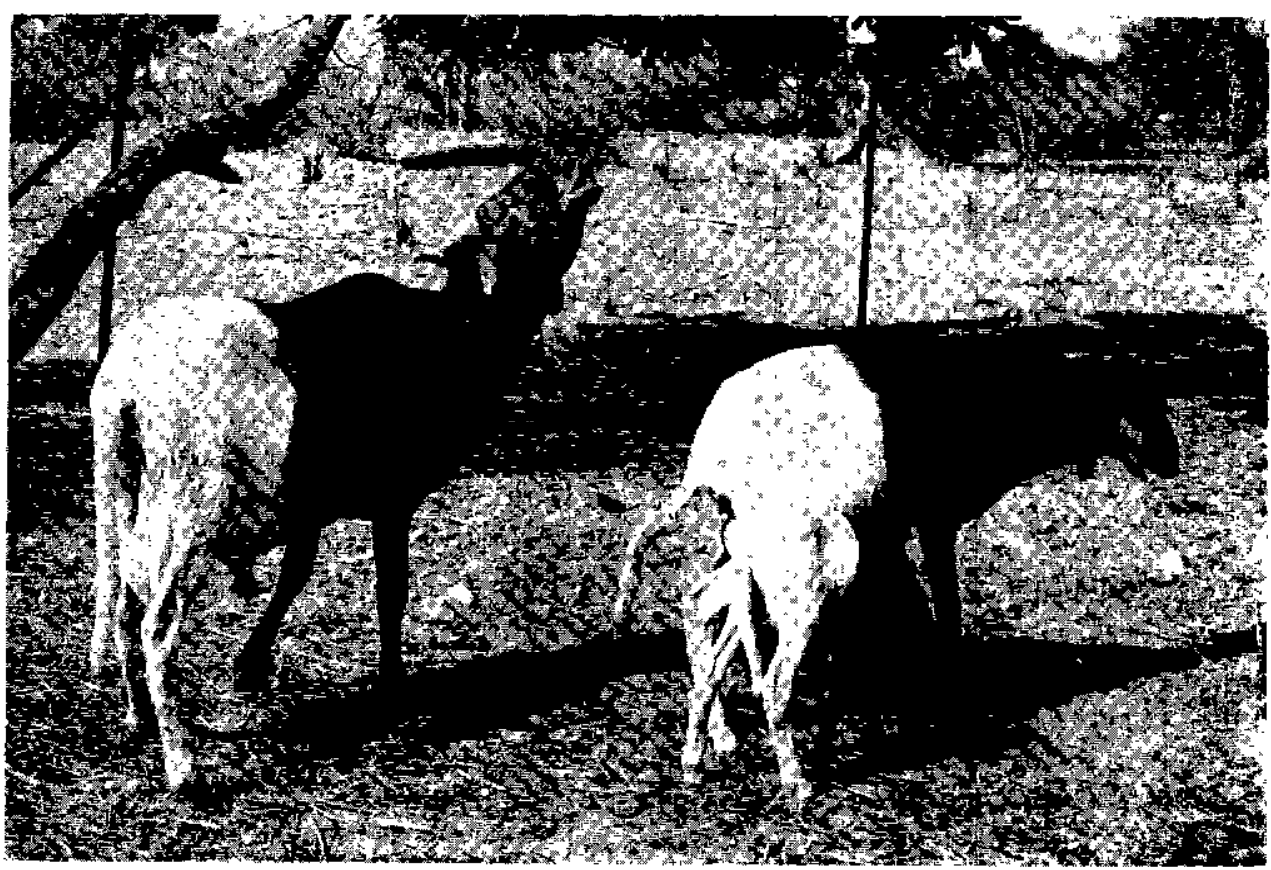

Photo I. - Le bélier effectue un «Flehmen» après avoir flarré l'urine de la femelle. 
grands cercles à travers le troupeau, mais l'attendant ou même se retournant vers lui s'il ne la suit plus.

Ensuite ces fuites se transforment en simples dérobades, la brebis fait quelques rapides foulées en avant à l'approche du mâle, puis l'attend en le regardant, la tête retournée vers l'arrière (Photo II).

Enfin apparaissent ce que nous appelons les "petits cercles»: en réponse aux avances du bélier, la brebis ne fuit plus mais se retourne brusquement sur elle-même, empêchant le mâle de se

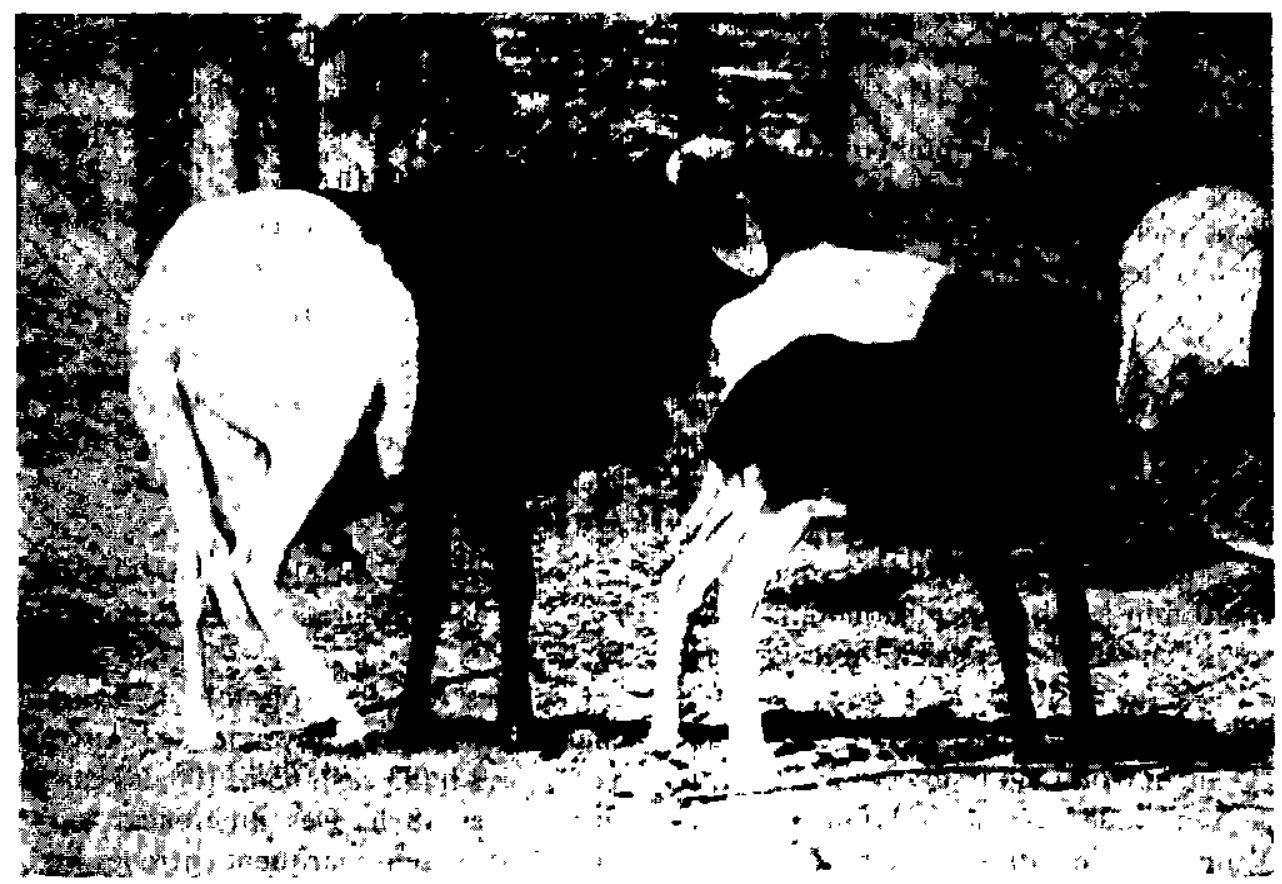

Photo II. — Parade sexuelle : la brebıs fixe le bélıer, la tête retournée vers l'arrière.

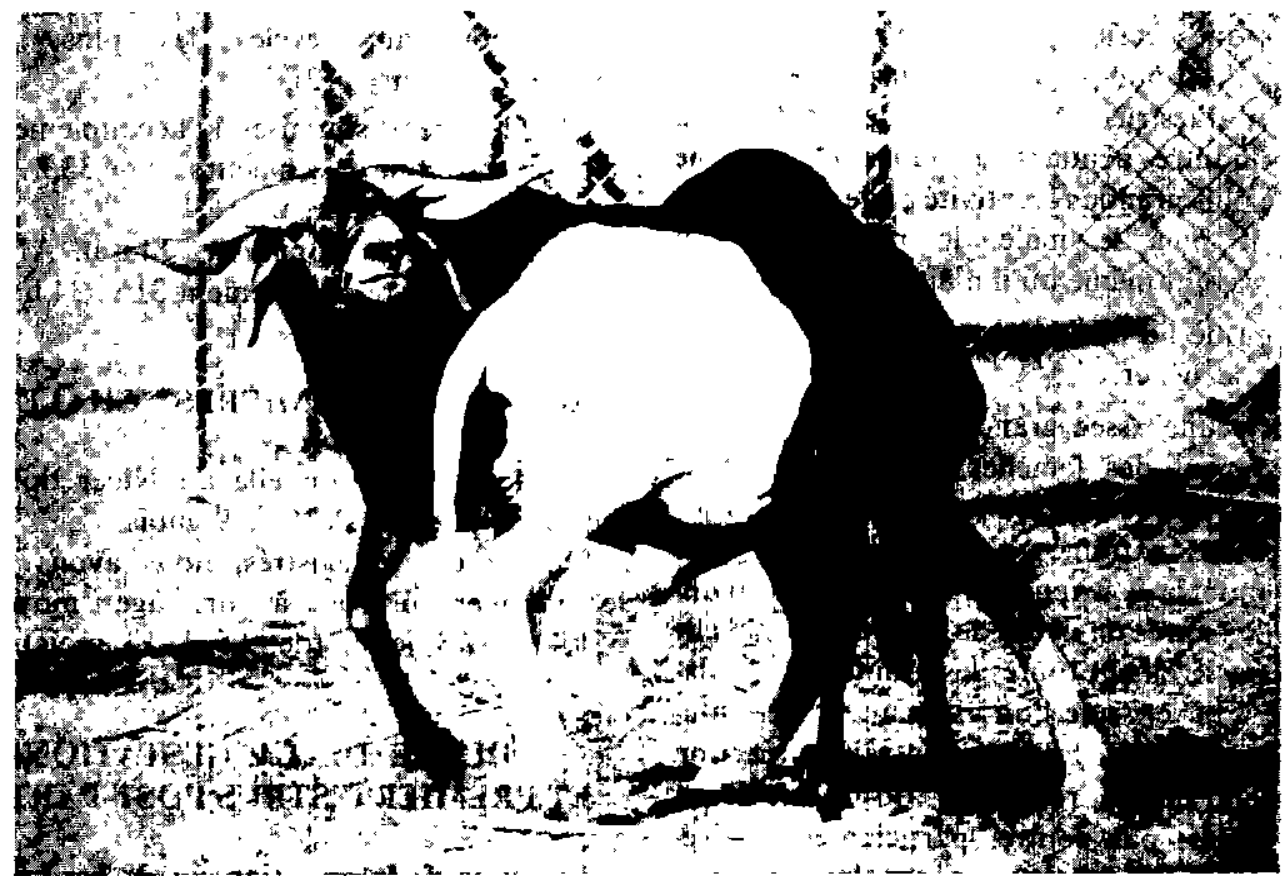

Photo III. - Parade sexuelle : position parallèle tête-bêche pendant les « petits cercles ». 
placer derrière sa croupe. Il en résulte une succession de petits cercles où les 2 animaux tournent sur eux-mêmes dans une position parallèle têtebêche. A ce stade, la femelle se laisse fréquemment flairer la sphère génitale en écartant légèrement la queue. Lorsque le bélier est peu actif la brebis tourne la tête vers lui, lui flaire le cou, les flancs ou le pénis et lui donne parfois de petits coups de la tête et du museau, semblant l'inciter à reprendre sa cour (Photo III).

Dans un dernier stade la brebis s'immobilise de plus en plus nettement devant les avances du mâle, lui permet de rester derrière elle et enfin accepte les montes qui aboutiront rapidement au coït.

Après une monte suivie d'intromission et d'éjaculation, les 2 animaux restent quelques secondes immobiles côte à côte, la brebis tenant la queue arquée à la base, décollée de la vulve. Parfois elle flaire ensuite le pénis du bélier, puis broute. La position typique de la queue est conservée $10 \mathrm{~min}$ à $1 \mathrm{~h}$ et peut donc ainsi sous l'effet de coïts successifs persister toute la durée de l'œstrus. Cette position de la queue, parfois citée comme typique de l'œstrus, n'est qu'une réaction à une intromission suivie d'éjaculation. On ne la remarque pas chez les femelles soumises au contrôle d'œstrus, ni même après une monte suivie d'intromısion sans éjaculation.

Quelques minutes après le coït, le bélier reprend sa cour. La brebis recommence alors le cycle typique décrit : fuites intenses, dérobades, petits cercles, immobilité, cela surtout en début et en fin d'œstrus. Au milieu de l'œstrus, la femelle montre pendant quelques heures une phase de plus grande réceptivité ; elle ne fuit pratiquement plus le mâle, le recherchant au contraire avant même qu'il n'ait récupéré.

A la fin de l'œstrus, la brebis fuira systématiquement le bélier.

Il existe une assez grande variabilité dans le comportement des femelles : si la majorité se comporte typiquement comme décrit ci-dessus, quelques cas extrêmes s'en distinguent: une femelle montra à 2 reprises une véritable nymphomanie : après un début de chaleur normal elle accepta passivement toutes les montes et copulations de 2 béliers que l'on alternait, et cela plusieurs jours durant. Elle ne montrait ni réaction d'évitement, ni de recherche du mâle. Deux autres brebis, par contre, participaient activement aux comportements précopulatoires mais se dérobaient systématiquement aux montes.

\section{CARACTÉRISTIQUES}

\section{DU CYCLE GESTRIEN ET DE L'GSTRUS}

\section{Longueur du cycle astrien}

Sur 86 cas contrôlés, nous obtenons une moyenne de $17,77 \pm 0,28$ jours. Des différences individuelles faibles mais significatives sont notables ; nous donnons ci-après les cas extrêmes :

- Brebis aux cycles les plus courts : $16,6 \pm 0,4$ jours $(n=12)$;

- Brebis aux cycles les plus longs: $18,3 \pm 0,7$ jours $(\mathrm{n}=16)$.

Ces résultats sont très voisins de la durée du cycle chez d'autres races tropicales : ANDERSON (1) donne 17,5 jours chez les brebis Masaï et 17,3 jours chez les brebis des Somalis.

\section{Durée de l'astrus}

Nous considérons ici la durée entre la première et la dernière monte acceptées par une brebis.

Chez les femelles soumises aux contrôles de chaleur, la durée moyenne de l'œstrus est de $42,6 \pm 1,9$ h ( 93 cas), 65 p. 100 des cas se situant entre 38 et $48 \mathrm{~h}$. Des différences individuelles significatives se remarquent entre les brebis, avec les 2 extrêmes suivant :

- Brebis aux cestrus les plus longs : $52,6 \pm 5,2 \mathrm{~h}(\mathrm{n}=15)$;

- Brebis aux cycles les plus courts : $33,3 \pm 7,9 \mathrm{~h}(\mathrm{n}=12)$.

Chez les brebis soumises à l'accouplement réel, la durée moyenne des chaleurs est de 33,2 $\pm 3,1 \mathrm{~h}$ ( $\mathrm{n}=28$ ).

Chez les brebis Somalis et Masaï, ANDERSON (1) donne respectivement 31 et $34 \mathrm{~h}$.

\section{AGE AUX PREMIERES CHALEURS}

L'opinion traditionnelle au Niger donne un âge à la puberté de 7 à 9 mois.

Sur 12 cas enregistrés, nous avons obtenu le premier œstrus à un âge moyen de $351,4 \pm 37,8$ jours (de 9,5 à 14 mois).

\section{DURÉE DE LA GESTATION ET PREMIER GSTRUS POST-PARTUM}

La durée de la gestation sur 28 cas contrôlés est de $154,8 \pm 1,0$ jours. 
Le premier œstrus post-partum survient $61 \pm 9$ jours après la mise bas, avec une assez large variation, entre 41 et 104 jours. Pour 8 cas, nous avons observé un cestrus silencieux avant le premier œstrus effectif.

\section{NOMBRE DE JEUNES PAR PORTÉE}

La taille moyenne par portée est de $1,1 \pm 0,1$ $(n=28)$ jeunes.

\section{SAISON DE REPRODUCTION}

Sur 136 astrus contrôlés, il n'apparaît aucune variation annuelle dan's la fréquence des chaleurs. La brebis Oudah est propre à se reproduire toute l'année (Graphique I).

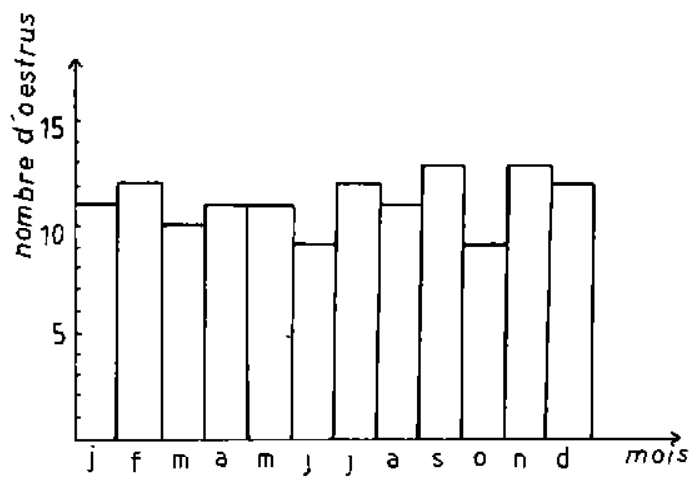

Graphique 1. Répartition mensuelle des 136 oestrus observés en cours d’année

Dans les troupeaux nomadisant du Niger, on observe par contre une période de chaleurs silencieuses en avril-mai (2) peut-être dues au déficit alimentaire et aux hautes températures que les animaux subissent à cette époque.

Chez toutes les races de moutons des pays tempérés ou froids, il existe une saison de reproduction bien marquée : cette saison est due à un anœstrus saisonnier des brebis réglé par les variations photopériodiques et elle permet aux animaux de mettre bas à une époque favorable à l'élevage des jeunes (MAULEON et ROUGEOT 1962, ANDERSON 1972, HAFEZ 1950/51, HUNTER 1964, SAHANI et al., 1976, WHEELER et LAND 1977, EL WISHY et al., 1976). Il existe une relation directe entre la durée de cette saison et la latitude, de même que l'altitude des régions originelles des races considérées; elle est d'autant plus courte que les races proviennent d'une région plus au nord et plus élevée (11). Ce caractère a complètement disparu (ou n'a jamais existé) chez les races tropicales : cas du Oudah, du mouton des Somalis et du mouton Masaï (1), ainsi que de différentes races indiennes (14). Même lorsqu'ils vivent à des latitudes élevées, ces moutons continuent à se reproduire toute l'année, ainsi que l'on peut le voir par exemple au zoo de Munich (Allemagne) pour une race du Cameroun et pour le mouton à tête noire.

Un phénomène identique se retrouve chez les chèvres (4).

\section{CONCLUSION}

La brebis Oudah recevant une nourriture régulière tout au long de l'année met bas tous les 7 mois une portée de 1,1 jeunes et peut donc produire 2 agneaux par an. Dans les conditions d'élevage nomade traditionnel, la fécondité est beaucoup plus basse à cause d'anœstrus ou de chaleurs silencieuses dus au déficit alimentaire en saison sèche et par la difficulté pour une brebis d'élever des jumeaux en dehors des quelques mois de l'année où la nourriture est abondante.

Une comparaison rapide entre quelques races tropicales, en particulier le Oudah, et les brebis à laine européennes montre les caractéristiques suivantes :

- Absence de saison de reproduction chez les races tropicales;

- Puberté nettement plus tardive chez la brebis Oudah (9,5 à 14 mois) que chez les brebis européennes (7 à 12 mois) $(5,9,6)$;

- La durée de la gestation, de même que la longueur du cycle astrien, sont du même ordre de grandeur ;

- La durée de l'œstrus par contre est nettement plus longue chez les races tropicales (Oudah, 42,6h, Somalis et Masaï respectivement 31 et $34 \mathrm{~h}$, brebis européennes 15 à $29 \mathrm{~h})(3,1,9,6)$.

\section{REMERCIEMENTS}

\section{L'auteur remercie vivement :}

- M. A. YENIKOYE, qui l'a aimablement autorisé à utiliser ses animaux pour certaines observations et les résultats de ses contrôles d'œstrus ;

- M. G. NOUHOU, garçon de laboratoire et M. BOUBACAR, berger, pour l'aide efficace qu'ils lui ont accordée tout au long de son travail. 


\section{SUMMARY}

\section{Reproduction characteristics of Oudah ewe}

From January 1977 until June 1979, the reproduction characteristics in a small flock of Oudah sheep at the University of Niamey (Niger) were studied. The sexual behaviour of the female is described as well as the principal results concerning the cestrus (length of cycle, duration of cstrus, anual distribution), the time of gestation and the reproduction rate.

\section{RESUMEN}

\section{Características de la reproducción de la oveja Oudah}

Se estudió las características de la reproducción de la oveja de raza Oudah en un pequeño rebaño en la Universidad de Niamey, Niger, de enero 1977 a junio 1979. Se describe el comportamiento sexual de la hembra $y$ los principales resultados referentes al estrus (duración del ciclo, duración del estrus, repartición anual), a la duración de la gestación y al número de crías por camada.

\section{BIBLIOGRAPHIE}

1. ANDERSON (J.). The estrus cycle in Merinos, Masai and Somali Sheep. Zootech. Vet., 1972, 27 (3-4) : 57-72.

2. ARI (Ibrahim Toubo). Contribution à l'étude de I'élevage ovin au Niger. Thèse Méd. vét., Dakar, 1975.

3. BANKS (E.). Some aspects of sexual behaviour in domestic sheep, Ovis aries. Behaviour, 1964, 23 : 249-279.

4. DEVENDRA (C.), BURNS (M.). Goat production in the tropics. Edinburgh, R. and R. Clark 1td, 1970.

5. DYRMUNDSSON (1972-1973). In : VAISSAIRE (1977).

6. EDNEY (T. N.), KILGOUR (K.), BREMMER (K.). Sexual behaviour and reproductive performance of ewe lambs at and after puberty. $J$. agric. Sci., 1978, 90 (2) : 83-91.

7. EL WISHY, EL SAWAF, EL MIKKAWI. Some aspects of reproduction in fat-ta1led sheep in subtropics : I. Reproductive behaviour of local Ausimi and imported Awasi ewes. Vet. med. J., Cairo Univ., 1971, 19 : 131-155.

8. EL WISHY, EL SAWAF, FOUAD. Some aspects of reproduction in fat-tailed sheep in subtropics: II. Seasonal variation in ovarian activity $Z$. Tierzüchtg. Züchtungsbiol., 1976, 93 (2) : 121-134.

9. FRASER (A. F.). Reproductive behaviour in Ongulates. London, New-York, Academic Press, 1968.
10. HAFEZ (E. S. E.). Sexual season of the ewe and daylight environment. Nature, 1950, 166: 822-823.

11. HAFEZ (E. S. E.). Die Brunftzeit bei Schafen in Beziehung zur Tageslänge in nordliche und südliche Breite. Naturwissenschaft, 1951, 38 : 100-110.

12. HUNTER (G. L.). The effects of season and mating on cestrus and fertility in the ewe. Proc. s. afr. anim. Prod. 1964, 3 : 195-206.

13. MATTNER (P. E.), BRADEN, TURNBULL. Studies in flock mating of sheep ; I. mating behaviour. Aust. J. exp. Agric. anim. Husb., 1967, 7 : 103-109.

14. SAHANI (K. L.), TIWARI (S. B.), SAHANI (M S.). Effect of the season on occurence of cestrus and fertility in different breeds of sheep under semi-arid conditions. Ind. vet. J., 1976, 53 (7) : 515-522.

15. SAMBRAUS (H. H.). Das Sexualverhalten der domestizierten einheimischen Wiederkäuer. Z. Tierpsychol., 1973 Beiheft 12.

16. VAISSAIRE (J. P.). Sexualité et reproduction des mammifères domestiques et de laboratoire. Paris, Maloine, 1977.

17. WHEELER, LAND. Seasonal variation in cestrus and ovarian activity of finnish Landrace, tasmanian merino and scottish blackface ewes. Anim. Prod., $1977,24: 363-376$.

18. YENIKOYE (A.), ANDRE (D.). Durées du cycle cestral, de l'œestrus et niveau de progestérone plasmatique au cours du cycle ostral chez la brebis peuhl du Niger (sous presse). 